\title{
Quimioterapia en cáncer de próstata ¿mito o realidad?
}

\author{
L.A. Rioja Sanz \\ Servicio de Urología. Hospital Universitario Miguel Servet. Zaragoza. \\ Unidad de Cáncer de Próstata. Dres. Allepuz, Gil Sanz, Gil Martínez y Borque. \\ Actas Urol Esp 2005; 29 (8): 723-724
}

$\mathrm{E}^{\mathrm{n}}$ n el pasado Congreso de la A.E.U. de San Sebastián, se programó y desarrolló, en la sesión plenaria del día 28 de mayo, un debate que intentó aclarar ante los asistentes, los puntos de vista, evidencias, e incertidumbres sobre las indicaciones de la quimioterapia en el cáncer de próstata en estos momentos, bajo la lectura de un urólogo el Prof. Sánchez Chapado, de un oncólogo, el Prof. Guillém Porta del IVO y la moderación que me fue encomendada.

El Director de Actas Urológicas, Dr. J.L. Ruiz Cerdá, me invitó a escribir un editorial sobre este tema, y en coincidencia sobre su actualidad, asumo responsablemente.

En la década de los hoy lejanos 80'-90', el carcinoma avanzado de próstata, en la evolución clínica y datos radiológicos y ¿biológicos? de aquellos momentos se convertía en hormonorrefractario, en situaciones próximas al final de la vida de esos pacientes, casi siempre en un contexto de obstrucción de tracto urinario inferior, afectación del superior, insuficiencia renal, disminución de los mínimos indicadores de calidad de vida, y llevábamos al paciente cálidamente, con la terapia paliativa, al final, en la mejor dignidad del ser doliente.

El descubrimiento y generalización del PSA, con su influencia en los programas de diagnóstico precoz, y diseño de pautas de screening, cambió espectacularmente los porcentajes de pacientes localizados que diagnosticábamos los urólogos en detrimento feliz de los localmente avanzados y diseminados ${ }^{1,2}$.

Ello llevó implícitamente al aumento de indicaciones de indicación curativa, y en el otro extremo, al reconocimiento de pacientes, en los que el nuevo marcador mostraba signos de hormonorrefractariedad (por su progresión) antes de que los signos clínicos clásicos de dolor por extensión de las metástasis, radiológicos, o de elevación de las fosfatasas ácidas, nos indicaran la situación de Hormonorrefractariedad (HRPC) y progresión, que eran nuestra guía en los 80-90. Cambio espectacular de escenario clínico.

Surgió racionalmente el concepto de $2^{\mathrm{a}}$ línea de tratamiento ${ }^{3}$ y el diseño de esquemas de agentes quimioterápicos en esta situación.

Pero la revisión que en 1993 realizó Yagoda ${ }^{4}$ de 26 ensayos realizados entre 1.988 y 1991 , incluyendo más de 1.000 pacientes, con unos rangos de respuesta del $8,7 \%$ y unos periodos de supervivencia entre 6-10 meses al convertirse en HRPC, la conclusión a la que se llegó es que era tóxica e inefectiva. Esta filosofía fue reforzada en el $2001^{5}$ por el análisis sistemático de 52 ensayos que integraban a 2.028 pacientes.

En ese escenario y momento, hubo dos publicaciones $^{6,7}$ que mostraban el beneficio clínico, pero sin mejora en la supervivencia, que experimentaban los pacientes en (HCRP) con el uso combinado el Mitoxantrone y Prednisona. Los estudios de Tannock ${ }^{6}$ y Kantoff ${ }^{7}$ permitieron la adopción de este esquema como el "Tratamiento Estándar" en esta situación.

Ocho y cinco años después de la publicación de estos artículos, (1996 y 1999), la comunidad urológica y los oncólogos nos vimos impactados por la publicación de dos ensayos en fase III multicéntricos y aleatorizados, que mostraban los efectos de los taxanos en el cáncer de próstata hormonorrefractario ${ }^{8,9}$ y nada más ni nada menos que en New England Journal of Medicine.

Estos estudios mostraban no sólo mejorías en los rangos de PSA y medidas de la calidad de vida, sino en la supervivencia. Y ha sido en estos estudios donde se ha demostrado por primera vez esta mejora de supervivencia, pero debemos mirar con cautela e ilusión estos resultados antes de concluir que todos los pacientes HRPC deben ser tratados con este agente. 


\section{¿Por qué?.}

Por las dos lecturas que pueden hacerse de los resultados, tanto el estudio (TAX 327), como el (SWOG 99-16) demuestran mejorías estadísticamente significativas en supervivencia, disminución PSA, tiempo de progresión y calidad de vida. El "vaso medio vacío" muestra que los taxanos son activos en el $40 \%$ de los pacientes, la supervivencia es limitada (aún siendo estadísticamente significativa). Mediana de 2,4 meses. Los nuevos esquemas tienen sólo un $9 \%$ más de actividad que el Mitoxantrone, y en contrapunto adolecen de más toxicidad. Sin olvidar los aspectos económicos.

Por todo ello persisten dilemas, antes de poder categorizar que en todos los pacientes afectos de HRPC haya que usar quimioterapia con docetaxel.

Habrá que establecer criterios clínicos y biológicos que permitan identificar a los pacientes que puedan beneficiarse de esta terapia activa. En los criterios de inclusión de estos dos relevantes artículos, llama la atención, (y no es perfil del paciente español en hormonorrefractariedad) que más del $80 \%$ tienen un excelente estado general, y la mediana de edad está entre 68 y 69 años. iiAquí son un poco más mayores!!. Hubo una indudable "selección".

Y la investigación biológica, puede, como en el cáncer de mama, o en el de pulmón, identificar a posibles pacientes respondedores. Es el caso del uso de Trastuzumab (herceptin) en las pacientes Her 2 positivo $^{10}$ o del uso del Gefitinib en las mutaciones del epidermal grow factor en el cáncer de pulmón ${ }^{11}$.

Posiblemente, y es el "vaso medio lleno" con el que concluyo estos comentarios, estamos asistiendo al nacimiento de una nueva época en el tratamiento del cáncer avanzado de próstata.

Se contempla la evaluación de nuevos agentes y nuevas combinaciones.

Así como la elaboración de nuevas indicaciones en Neoadyuvancia y Adyuvancia.

$Y$ aunque en nuestro pensamiento clínico y quirúrgico, las nuevas estrategias del tratamiento del cáncer enfocadas en dianas moleculares, no son fáciles de seguir, tenemos la obligación de seguir asumiendo el enfoque integral del paciente con cáncer de próstata, transmitirlo a nuestros residentes, y contemplar en ese amanecer que significan los datos iniciales de la quimioterapia y las nuevas estrategias, la responsabilidad del urólogo y la oportunidad de darnos la mano con los oncólogos médicos en los momentos en que su experiencia y pericia puedan ayudar a nuestros pacientes.

No deberíamos caer en la simplificación de tratar al paciente con cáncer localizado, localmente avanzado y diseminado hasta que llegue a la hormonorrefractariedad. Y en ese momento confiárselo al oncólogo y dejar el control de la evolución.

El momento actual, en la responsabilidad de la Urología Española con nuestros pacientes, exige el rigor y sentido crítico para evaluar resultados "espectaculares" en su justo valor, así como seguir los avances de la investigación básica para incluirnos comprometidamente en los ensayos clínicos y seguir siempre dando la mano al paciente que no va bien (o que sigue el camino natural de la enfermedad) y un día confió en nosotros.

\section{REFERENCIAS}

1. Farkas A., Scheneider D., Perrotti M., Cummings K.B. Ward W.S National trends in the epidemiology of prostate cancer, 1973 to 1994 evidence for the efectiveness of prostate-specific antigen screening. Urology, 1998;52:444-449.

2. Svetec D. Thompson I.M. PSA screening - current controversy. Ann Oncol. 1998;9(12): 1283-1288.

3. Schröeder FH Total androgen suppression in the management of prostatic cancer. A critical review. In Schröeder FH, Richards B (ed) Progress in Clinical and Biological Research, vol 185 A. EORTC GU Group Monograph 2, Part A. Therapeutic Principles in Metastatic Prostatic Cancer. New York, Alan R. Liss 1985,307-317.

4. Yagoda A. Petrylak D. Cytotoxic chemotherapy for advanced hor mone-resistant prostate cancer. Cancer 1993;71:1098-1109.

5. Casciano R, Petrylak D. Systematic review of chemotherapy efficacy from controlled trials in hormone refractory prostate cancer patients. Proc Am Soc Clin Oncol 2001;20:2428.

6. Tannock IF, Osoba D, Stockler MR, Ernst DS, Neville AJ, Moore MJ et al. Chemotherapy with mitoxantrone plus prednisone or prednisone alone for symptomatic hormone resistant prostate cancer: a Canadian randomised trial with palliative end points. J Clin Oncol 1996;14:1764- 765.

7. Kantoff PW, Halabi S, Conaway M, Picus J, Kirshner J, Hars V et al. Hydrocortisone with or without mitoxantrone in men with hormone refractory prostate cancer: results of the cancer and leukaemia group B 9182 study. J Clin Oncol. 1999 Aug; 17(8):2506-513.

8. Tannock IF, de Wit R, Berry WR, Horti J, Pluzanska A, Chi KN, et al. Docetaxel plus Prednisone or Mitoxantrone plus Prednisone for Advanced Prostate Cancer. N Engl J Med. 2004 Oct 7;351(15): 15021512.

9. Petrylak DP, Tangen CM, Hussain MH, Lara PN Jr, Jones JA, Taplin ME, et al. Docetaxel and Estramustine Compared with Mitoxantrone and Prednisone for Advanced Refractory Prostate Cancer. N Engl J Med. 2004 Oct 7;351(15):1513-1520.

10. Jones RL. Smith IE. Efficacy and safety of trastuzumab. Expert Opin Drug Saf 2004;3(4):317-327.

11. Paez JG, Janne PA, Lee JC, Tracy S, Greulich H, Gabriel S et al. . EGFR Mutations in lung cancer: correlation with clinical response to Geftinib therapy. Science 2004;304:1497-1500.

Prof. L.A. Rioja Sanz

Po Constitución 25

50001 Zaragoza 